\title{
NEW DEVELOPMENTS OF PHOTODETECTORS FOR THE LAKE BAIKAL NEUTRINO EXPERIMENT
}

\author{
B. K. LUBSANDORZHIEV \\ Institute for Nuclear Research of Russian Academy of Sciences, pr-t 60-letiya \\ Oktyabrya 7 A, \\ Moscow 117312, Russia \\ E-mail: lubsand@pcbai10.inr.ruhep.ru
}

\begin{abstract}
New developments of photodetectors for the lake Baikal neutrino experiment are described. Some test results of photodetectors at the lake Baikal are presented.
\end{abstract}

\section{Introduction}

The lake Baikal Neutrino Experiment has history of more than 20 years, starting from small short experiments with a few PMTs in the early 80s to the present large scale longterm operating neutrino telescope NT-20d, which has been put into operation on April 6th 1998. The telescope's effective area for muons is 2000-10000 depending on a muon energy. The rate of events due to atmospheric neutrinos is about 1 per two days.

\section{Neutrino telescope NT-200}

The lake Baikal Neutrino Telescope NT-200 is located in the southern part of the great Siberian lake Baikal at $3.6 \mathrm{~km}$ from the shore and at the depth of $1 \mathrm{~km}$. The schematic view of NT-200 and optical modules attached at a string are presented in fig.1. The telescope consists of 192 optical modules at 8 vertical strings arranged at an umbrella like frame. Optical modules are grouped in pairs and swithched in coincidence with $15 \mathrm{~ns}$ time window. So two optical modules in one pair define one optical channel resulting in rather low optical channel background counting rate of $100-300 \mathrm{~Hz}$. Two pairs of optical modules form so called svyazka. The detector electronics system is hierarchical: from the lowest level to the highest one - optical module electronics, svyazka electronics module, string and detector electronics modules. In the latter detector trigger signals are formed and all information from string electronics modules are received and sent to the shore station. Three underwater electrical cables connect the detector with the shore station. The detector is operated from the shore station. 


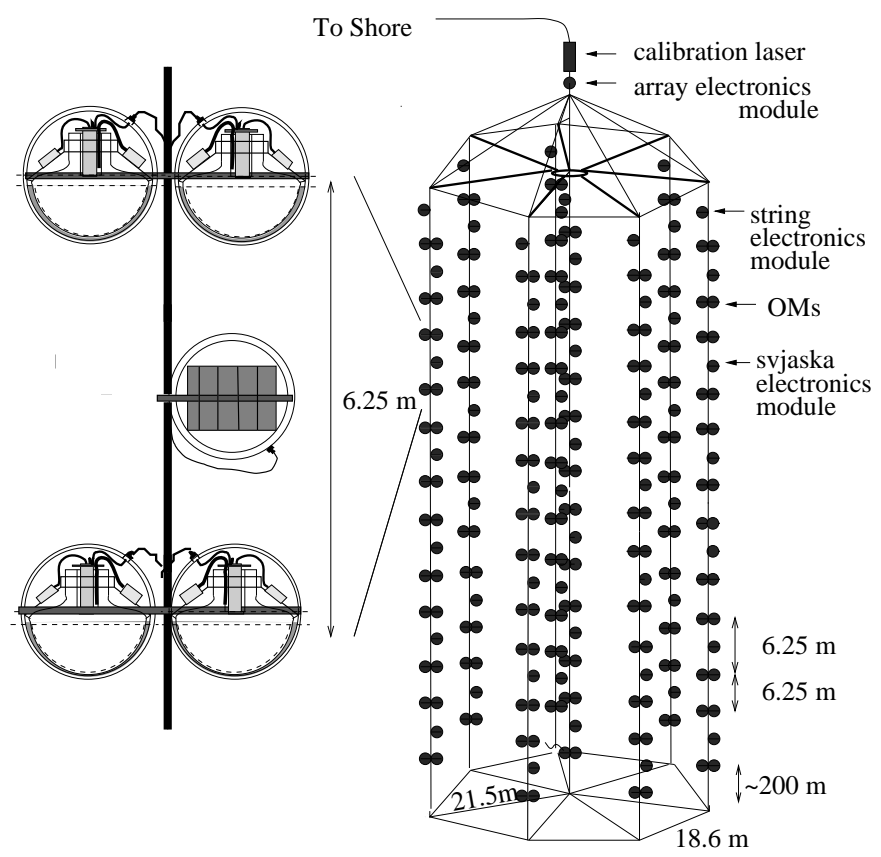

Figure 1: The lake Baikal neutrino telescope NT-200

\section{Quasar-370 phototube}

The Quasar-370 phototube国毗 is a hybrid phototube which consists of an electro-optical preamplifier with large area hemispherical photocathode and small conventional type PMT, see fig.2. Photoelectrons from a large $37 \mathrm{~cm}$ diameter hemispherical photocathode with $2 \pi$ acceptance are accelerated by 25 $\mathrm{kV}$ to a fast, high gain luminescent screen ( $Y S O$ scintillator is usually used). The light flashes in luminescent screen induced by photoelectrons are read out by small PMT with $3 \mathrm{~cm}$ diameter photocathode. The latter has been developed especially for this kind of application by INR and MELZ factory 6 . As a result one photoelectron from the hemispherical photocathode yields typically 25 photoelectrons in the small PMT.This high gain first stage results in an excellent single photoelectron resolution. Due to the fast acceleration of primary photoelectrons by $25 \mathrm{kV}$ high voltage and mushroom shaped glass envelope the time jitter can be kept rather low. Last but not the least the tube is almost insensitive to the Earth's magnetic field. Averaged over more than 200 tubes the mean values for single photoelectron resolution and time 


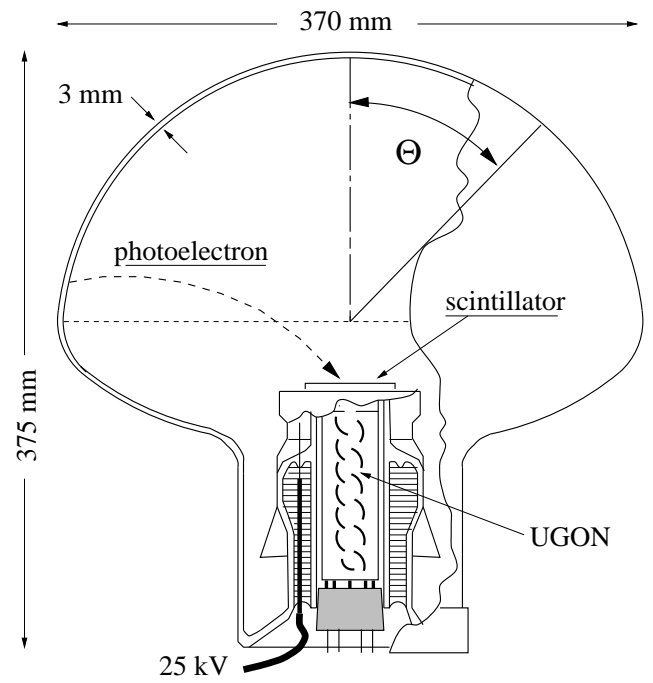

Figure 2: Quasar-370 phototube

resolution is $70 \%$ and 2 ns respectively. Quasar-370 phototube is characterized by conspicuously low level of afterpulses in comparison with conventional PMTs. The level of afterpulses in Quasar-370 tube is substantially less than $1 \%$. Another advantage of Quasar-370 phototube in contrary to conventional PMTs is the lack of prepulses due to the fact that the first stage of the phototube is optically separated from tube's photocathode. It was shown 2 B 3 - that Quasar-370 parameters depend strongly on characteristics of a scintillator in a luminescent screen. So recently we have developed a number of modifications of Quasar-370 tube with new scintillators. The most promising results we have got with $\mathrm{ScBO}_{3}, Y A P$ and $\mathrm{LSO}$. Unfortunately the latter one has one substantial drawback for using in Quasar-370 tube. It has rather high value of $Z_{\text {eff }}$. It is important to note here we need scintillators with $Z_{\text {eff }}$ as low as possible to suppress effectively late pulses 7 due to photoelectron backscattering effect. With these new scintillators we have reached 1ns (FWHM) time jitter and $40 \%$ single photoelectron resolution. It is noteworthy to mention here about very much intriguing scintillatol $-Z n O: G a$ with less than 1 ns decay time and light yield of $40 \%$ of $N a I: T l$. At present we try to work with this scintillator and to reproduce results of 6 . The success of this work would undoubtedly be a kind of a breakthrough in designing of very fast and effective hybrid phototubes. 


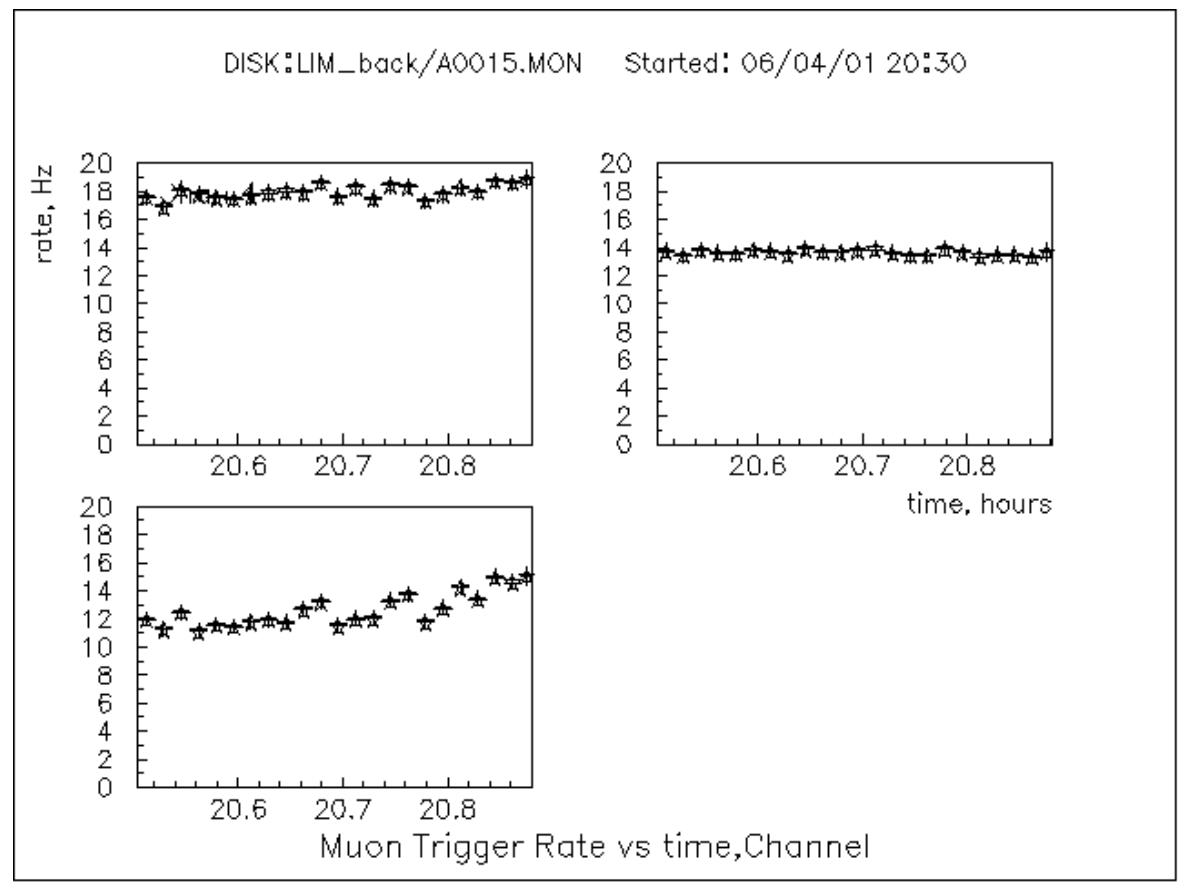

Figure 3: Muon Trigger rate versus time. Left up and left bottom - for conventional Baikal optical channel. Right up - for new optical channel based on new two-channel optical module.

\section{Two-channel optical module}

The pairwise ideology pursued in NT-200 neutrino telescope has many advantages. The ideology allows to suppress effectively an individual optical module background counting rate due to water luminescence and a phototube's dark current, to eliminate phenomena deteriorating phototube's time resolution namely prepulses, late pulses and afterpulses. Moreover such an approach facilitates very much designing of trigger system, data acquisition system etc. Unfortunately there is just one but essential shortcoming. Namely it's too expensive to have two optical modules for one optical channel. To overcome this problem we have developed two-channel optical module based on two-channel version of Quasar-370 phototubed.

Two-channel Quasar-370 phototube uses the same electro-optical preamplifier and new two-anode small PMT with mesh dynode system instead of conventional PMT. This new two-anode small PMT has following characteris- 
tics: time jitter $-\sigma=340 \mathrm{ps}$; Peak-to valley ratio of single photoelectron sharge distribution -1.5 ; cross-talks between channels $-1 \%$. Basing on two-channel Quasar-370 phototube we have developed two-channel optical module which incorporates besides Quasar-370 phototube two fast transimpedance preamplifiers for each anode signals and uses the same glass pressure vessel and the same penetrators as conventional Baikal optical module. In the course of the last expedition at the lake Baikal we have tested one pilot sample of two-channel optical module in frame of a new experimental string designed to test technological innovations for future neutrino telescopes at the lake. In the test measurements at the lake we used ordinary but slightly modified NT-200 front-end electronics. Output signals of each channel are switched in coincidence just in the same way as in NT-200. It results in the same local trigger counting rate of $100-300 \mathrm{~Hz}$. Fig.3 presents the time dependence of muon trigger rate for two conventional Baikal optical channels and new optical channel based on two-channel optical module. One can see that the new optical channel has comparable sensitivity to muons with ordinary optical channels of NT-200. It opens new possibilities for NT-200 further extension plans.

\section{Other developments}

A new version of Quasar-370 phototube (Quasar-370D) with semiconductor diode as a photoelectron multiplying element instead of a system of luminescent screen and small PMT has been developed. We have manufactured two pilot samples which are under studies now. Modifications of Quasar-370 phototubes (Quasar-370G)10 which are able to withstand high current due to night sky background operate successfully in the wide angle atmospheric air Cherenkov

detectors TUNKA11 and SMECA12. Quasar-370L is low background version of Quasar-370 phototube. It has $U^{238}$ and $T h^{232}$ content of about $10^{-8} \mathrm{~g} / \mathrm{g}$. Quasar-370L is aimed at using in low background experiments. Another development is concerned with phototubes with high quantum efficiency (more than $40 \%$ in the range $450-550 \mathrm{~nm}$ ) photocathodes and it's results will be reported elsewhere.

\section{Conclusion}

The lake Baikal Neutrino Experiment successful operation proves high performance and high reliability of a number of photodetectors developed for this kind of application. New developments are focused at future neutrino experiments at the lake Baikal and other experiments in high energy physics, cosmic ray and astroparticle physics. 


\section{Acknowledgments}

This work was supported by the Russian Ministry of Research (contract 10211(00)-p), the German Ministry of Education and Research and the Russian Fund of Basic Research (grants 99-02-1837a,01-02-31013 and 00-15-96794) and by the Russian Federal Program "Integration"(project 346). The author is indebted very much to his colleagues from KATOD and MELZ laboratoties and Baikal Collaboration. The author would like to thank particularly Dr. V.Ch.Lubsandorzhieva for many invaluable advices and help in preparation of this paper.

\section{References}

1. I.A.Belolaptikov et al, Astropart. Phys. 72631997

2. L.B.Bezrukov et al Proc. 3rd NESTOR Workshop, Pylos 1993. Univ.Athens. 1321994

3. R.I.Bagduev et al Proc. Int.Conf. "Trends in Astroparticle Physics", Teubner, Stutgart, 1321994

4. R.I.Bagduev et al, Nucl. Instrum. Methods A 420, 138 (1999)

5. B.K.Lubsandorzhiev, Nucl. Instrum. Methods A 442, 368 (2000)

6. L.B.Bezrukov et al, Instrum. and Experim. Techn. 11042000

7. B.K.Lubsandorzhiev et al, Nucl. Instrum. Methods A 442, 452 (2000)

8. PHILIPS Photomultiplier tubes, 6-35 1994

9. B.K.Lubsandorzhiev et al, Proc. of the 25th ICRC.7 2691997

10. B.K.Lubsandorzhiev et al, Instrum. and Experim. Techn. 31042001

11. N.Budnev et al, Proc. of the 25th ICRC.2 5812001

12. V.A.Balkanov et al, Yadernaya Fizika 6310272000 